\title{
Isolation, draft genome sequencing and identification of Enterobacter roggenkampii $\mathrm{CCl} 9$
}

\author{
Hironaga Akita ${ }^{\circledR}$, Yuya Itoiri², Noriyo Takeda ${ }^{1}$, Zen-ichiro Kimura ${ }^{2}$, Hiroyuki Inoue ${ }^{1}$, Akinori Matsushika ${ }^{1,3}$ \\ 1. Research Institute for Sustainable Chemistry, National Institute of Advanced Industrial Science and Technology (AIST), 3-11-32 Kagamiyama, \\ Higashi-Hiroshima, Hiroshima 739-0046, Japan. \\ 2. Department of Civil and Environmental Engineering, National Institute of Technology, Kure College, 2-2-11 Aga-minami, Kure, Hiroshima, 737-8506, Japan. \\ 3. Graduate School of Integrated Sciences for Life, Hiroshima University, 1-3-1 Kagamiyama, Higashi-Hiroshima, Hiroshima 739-8530, Japan. \\ $\triangle$ Corresponding author: Tel: +81 82-493-6848; Fax: +81 82-420-8291; E-mail: h-akita@aist.go.jp.
}

(C) The author(s). This is an open access article distributed under the terms of the Creative Commons Attribution License (https://creativecommons.org/licenses/by/4.0/). See http://ivyspring.com/terms for full terms and conditions.

Received: 2020.03.24; Accepted: 2020.09.05; Published: 2021.01.01

\begin{abstract}
Strain $\mathrm{CCl}$, which was isolated from leaf soil collected in Japan, was capable of growth on poor-nutrient medium, at temperatures of $10^{\circ} \mathrm{C}$ to $45^{\circ} \mathrm{C}$, at pHs of 4.5 to 10 , and in the presence of $7.0 \% \mathrm{NaCl}$. We determined a draft genome sequence of strain $\mathrm{CCl} 9$, which consists of a total of 28 contigs containing $4,644,734$ bp with a GC content of $56.1 \%$. This assembly yielded 4,154 predicted coding sequences. Multilocus sequence analysis (MLSA) based on atpD, gyrB, infB, and $r p o B$ gene sequences were performed to further identify strain CCI9. The MLSA revealed that strain CCI9 clustered tightly with Enterobacter roggenkampii $\mathrm{EN}-117^{\top}$. Moreover, the average nucleotide identity value (98.6\%) between genome sequences of strain $\mathrm{CCl}$ and $\mathrm{E}$. roggenkampii $\mathrm{EN}-117^{\top}$ exceeds the cutoff value for prokaryotic subspecies delineation. Therefore, strain $\mathrm{CCl9}$ was identified as $E$. roggenkampii $\mathrm{CCl9}$. To clarify differences between E. roggenkampii $\mathrm{EN}-117^{\top}$ and $\mathrm{CCl}$, the coding proteins were compared against the eggNOG database.
\end{abstract}

Key words: Enterobacter, Oligotroph, $16 \mathrm{~S}$ rRNA, draft genome sequence, multilocus sequence analysis, average nucleotide identity

\section{Introduction}

Microbial bioproduction enables continuous production of desired substances from nutrients including sugars, nitrogens and minerals. In the present day, therefore, a variety of microorganisms are used industrially for production of enzymes [1], medicines [2], and organic acids [3]. These microorganisms require high-nutrient media containing large amounts of sugar, nitrogen, phosphorus, mineral and other nutrients to optimize their productivities. On the other hand, the reduction of nutrient costs is important for achieving cost-effective production. Consequently, various attempts to reduce the production cost have been studied intensively in recent years. In general, the development of metabolically engineered strains with improved productivity of target substances is one of the most effective ways to reduce production costs [3]. For example, an engineered Mannheimia succiniciproducens strain is shown to increase the productivity of succinic acid and to decrease the by-product formation [4]. The utilization of agro-industrial wastes (such as sugarcane bagasse, potato peel waste and oil cake) as the nutrient sources also helps to reduce production costs [5].

Apart from those attempts, we expect that production costs could be reduced if an oligotrophic microorganism is used for industrial bioproduction, since oligotroph can grow under low-nutrient conditions. However, the doubling time of oligotroph is relatively long, and the growth of an obligate oligotroph is inhibited in the presence of high levels of nutrients [6]. These limitations make oligotroph difficult to use for the industrial bioproduction. Thus, new oligotrophic strain with fast growth rates is 
needed. Here, we report the isolation of an oligotroph, strain CCI9, with a fast growth rate. To facilitate future in-depth genomic studies and industrial applications of this strain, strain CCI9 was subjected to genome sequencing.

\section{Materials and Methods}

Soil samples were collected from HigashiHiroshima city in Hiroshima prefecture, Japan. A $1.5 \%$ agar plate ( $\mathrm{pH}$ 7.2) without any additional carbon and nutrient sources was used for isolation of the objective oligotroph. Agar (Nacalai tesque, Kyoto, Japan) used in this study contains sulfate $(<0.4 \%)$, calcium $(<0.1 \%)$, iron, $(<0.01 \%)$ and a few fatty acids and/or other minerals at concentrations less than $0.01 \%$. The $\mathrm{pH}$ of agar plate was adjusted using $\mathrm{NaOH}$. To isolate the oligotroph from the soil samples, soil samples were suspended at $10 \%(\mathrm{w} / \mathrm{v})$ with sterilized water, and the suspension was then filtrated to remove the soil sample. Subsequently, the filtrate was inoculated onto the plate. After the plate was incubated overnight at $37^{\circ} \mathrm{C}$, individual colony was successively re-streaked onto fresh $1.5 \%$ agar plates at least three times to obtain a pure colony.

After strain CCI9 was pre-grown overnight, the pre-culture was diluted to $\mathrm{OD}_{600}=0.05$ with fresh medium, the culture was incubated under several conditions. The $\mathrm{OD}_{600}$ was measured by monitoring the difference between cellular and cell-free turbidity values using an Eppendorf BioSpectrometer (Eppendorf, Hamburg, Germany). Growth of strain CCI9 was tested using Nutrient Broth (Kyokuto, Tokyo, Japan) at various temperatures $\left(5-50^{\circ} \mathrm{C}\right)$ and pHs (4.0-11.0), and in the presence of various concentrations of $\mathrm{NaCl}(0-8.0 \%, \mathrm{w} / \mathrm{v})$.

To prepare genomic DNA, strain CCI9 was cultured aerobically for $6 \mathrm{~h}$ at $37^{\circ} \mathrm{C}$ in Nutrient Broth (Kyokuto, Tokyo, Japan), and the cells were harvested by centrifugation. Extraction of genomic DNA from the cells was performed using an illustra ${ }^{\mathrm{TM}}$ bacteria genomicPrep Mini Spin Kit (GE Healthcare, Chicago, IL, USA) according to the manufacturer's instructions. The concentration and purity of the resulting genomic DNA were measured using a QuantiFluor ONE dsDNA System (Promega, Madison, WI, USA) and NanoDrop ND-1000 spectrophotometer (Thermo Fisher Scientific, Waltham, MA, USA), respectively.

Genome sequencing was performed using a MiSeq (Illumina, San Diego, CA, USA) sequencer. Default parameters were used for all software unless otherwise specified. The de novo assembly of the raw data was carried out using A5-miseq ver.20160825 [7]. The tRNA and rRNA genes were detected using Prokka v.1.14.0 [8]. Genome annotation was performed using Prokka v.1.14.0 [8] and the eggNOG- mapper website v.2.0.0 [9]. Average nucleotide identity (ANI) values were calculated by pairwise genome comparison of genome sequences of strain CCI9 and E. roggenkampii EN-117 $\mathrm{T}$ using the ANI algorithm [10] implemented within OrthoANIu tools [11]. The Venn diagram based on the predicted coding sequences was constructed using OrthoVenn2 [12]. To clarify differences between E. roggenkampii EN-117T and CCI9, the coding proteins were compared against the eggNOG database [9].

Multilocus sequence analysis (MLSA) was performed using the method of Brady et al. [13, 14]. A phylogenetic tree of concatenated sequences $(10,512$ $\mathrm{bp})$, including partial sequences of four housekeeping genes [atpD (encoding the $\beta$ subunit of ATP synthase; $1383 \mathrm{bp}$ ), gyrB (encoding DNA gyrase; $2412 \mathrm{bp}$ ), infB (encoding translation initiation factor 2; $2688 \mathrm{bp}$ ), and rpoB (encoding the $\beta$ subunit of RNA polymerase; 4029 bp)] from strain CCI9, was reconstructed using the maximum-likelihood method with the TamuraNei model [15]. The housekeeping genes of the related type strains were obtained from the GenBank/ DDBL/EMBL databases.

\section{Results and Discussion}

To obtain oligotrophs, filtrates were prepared from several soil samples such as compost, leaf soil, mud and peat moss. Subsequently, the filtrates were plated onto $1.5 \%$ agar $(\mathrm{pH} 7.2)$ without an additive carbon source or other medium components. After incubation overnight at $37^{\circ} \mathrm{C}$, a single colony was obtained from the filtrate of leaf soil, which is a soil composed mainly of decaying leaves. A purified colony was obtained through standard dilution plating on the same plates, and this isolate was designated strain CCI9 (strain number: HUT-8146). By contrast, Escherichia coli MG1655, which is used to create a bioproduction strain in many studies, did not grow on a $1.5 \%$ agar $(\mathrm{pH}$ 7.2). Although some oligotrophic bacteria are inhibited by high nutrient mixtures [16, 17], the growth of strain CCI9 was not inhibited in Nutrient Broth or LB medium. The growth rate of strain CCI9 was similar to that of E. coli MG1655, when cultured in Nutrient Broth or LB media (Fig. 1A). In Nutrient Broth, the strain CCI9 was capable of growing at temperatures of $10^{\circ} \mathrm{C}$ to $45^{\circ} \mathrm{C}$ (Fig. 1B) and at pHs of 4.5 to 10.5 (Fig. 1C), as well as showed the optimal growth at $30^{\circ} \mathrm{C}$ and $\mathrm{pH}$ 7.0. Moreover, this strain was tolerant to $7.0 \%(\mathrm{w} / \mathrm{v})$ $\mathrm{NaCl}$ (Fig. 1D). These results suggested that we had successfully isolated the desired oligotroph.

To determine the taxonomy of strain CCI9, we determined a draft genome sequence of strain CCI9. The raw data from the MiSeq yielded 5,879,580 reads with 218-fold coverage. The assembled genome 
sequence of strain CCI9 consisted of 4,644,734 bp with a GC content of $56.1 \%$. The 28 contigs in the assembly yielded the largest contig of 1,348,126 bp, and an N50 contig size of $283,167 \mathrm{bp}$. Within the genomic DNA of strain CCI9, 4,154 predicted coding sequences were identified, along with 83 tRNA genes and 11 rRNA genes.

As noted by Brady et al. [13, 14], MLSA is useful for determining the phylogeny of bacterial species. Therefore, we performed MLSA based on partial sequences of the $a t p D, g y r B$, infB, and $r p o B$ genes (Fig. 2 ). The resultant phylogenetic tree confirmed that strain CCI9 fell within a cluster comprising members of the genus Enterobacter. The most-closely related Enterobacter type strain was E. roggenkampii EN-117 [18], with $99.5 \%$ sequence similarity. The genome completeness values of strain CCI9 and E. roggenkampii EN-117T were 99.1\%. The ANI value between strain CCI9 and E. roggenkampii $\mathrm{EN}-117 \mathrm{~T}$ was $98.6 \%$, which exceeded the cutoff value of $98 \%$ for prokaryotic subspecies delineation [18]. Thus, strain
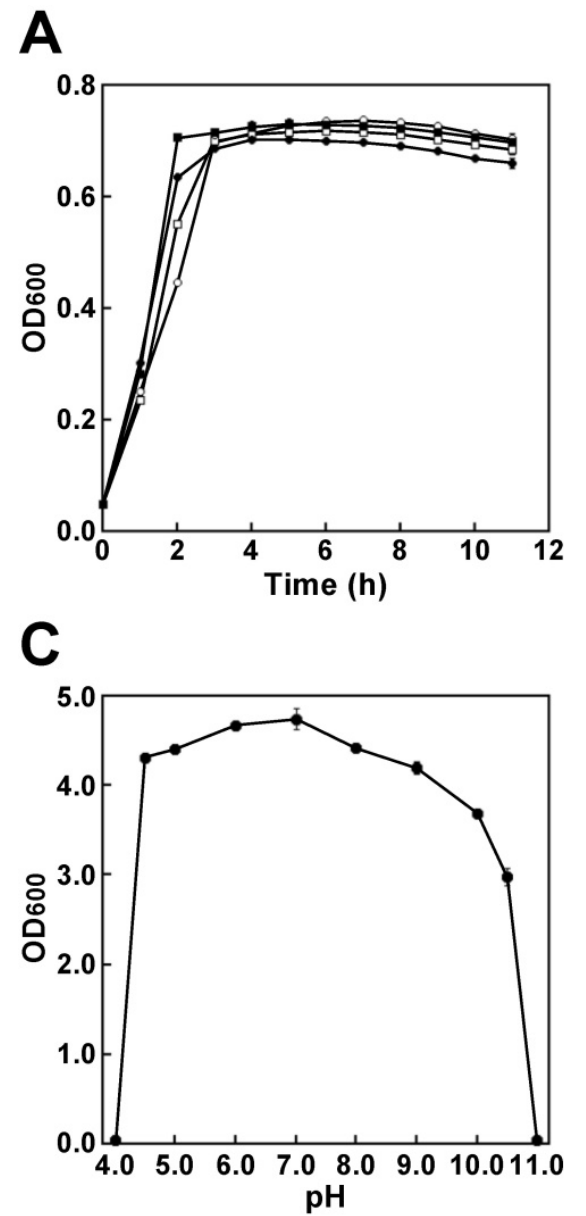

CCI9 was identified as E. roggenkampii CCI9.

The Venn diagram shows 3918 common clusters of genes that show high similarity between the two $E$. roggenkampii strains (Fig. 3). On the other hand, 273 and 236 unique genes were present in the draft genome sequences of E. roggenkampii $\mathrm{EN}-117^{\mathrm{T}}$ and $\mathrm{CCI}$, respectively. Among the eggNOG categories of both strains (Table 1), three kinds of genes involved in cell motility class (such as chemotaxis, fimbrial protein and outer membrane usher protein) were confirmed in the draft genome of E. roggenkampii CCI9. The motility properties of E. roggenkampii CCI9 may be different from that of E. roggenkampii EN-117T.

In this study, we described the isolation of strain CCI9, a bacterium capable of growth in nutrient-poor medium. After the draft genome sequence of strain CCI9 was determined, this isolate was identified as $E$. roggenkampii CCI9 based on MLSA and ANI value analysis. The draft genome sequence of $E$. roggenkampii CCI9 revealed their metabolic pathways, which is expected to use for the construction of

B

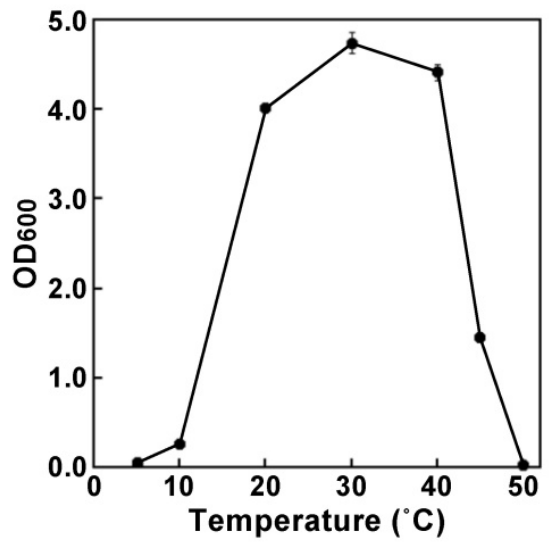

D

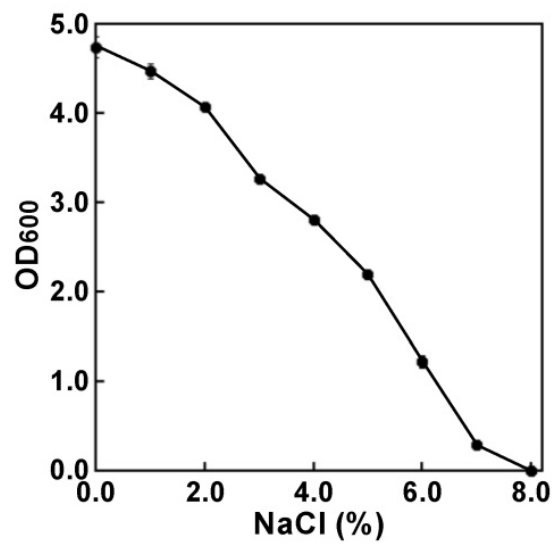

Figure 1. Phenotypic characterization of strain CCI9. (A) Growth ability at $37^{\circ} \mathrm{C}$ of strain $\mathrm{CCl9}$ and Escherichia coli MG1655. The results for strain CCI9 and E. coli MG1655 are shown as filled and open symbols, respectively. The media are indicated as follows: circles, Nutrient Broth (pH 7.0) and squares, LB media (pH 7.0). The $\mathrm{OD}_{600}$ was measured using a Bio Microplate Reader HiTS (Scinics, Tokyo, Japan). (B) Effect of culture temperature on the growth ability of strain CCl9. Strain CCl9 was cultured at $\mathrm{pH} 7.0$. (C) Effect of culture $\mathrm{pH}$ on the growth ability of strain $\mathrm{CCl9}$. Strain $\mathrm{CCl}$ was cultured at $30^{\circ} \mathrm{C}$. (D) Effect of $\mathrm{NaCl}$ concentration on the growth ability of strain CCI9. Strain CCI9 was cultured at $30^{\circ} \mathrm{C}$. Error bars indicate SE $(n=3)$. 
engineered derivatives. Indeed, metabolically engineered Enterobacter strains for the production of acetoin have been constructed by inactivation of the by-product pathways $[19,20]$.

\section{Nucleotide Sequence Accession Number}

The draft genome sequence of E. roggenkampii
CCI9 has been deposited in the DDBJ/EMBL/ GenBank databases under accession numbers BLPJ01000001 to BLPJ01000028. The raw sequence reads have been deposited in DDBJ under BioProject number PRJDB9407 and BioSample number SSUB014344.

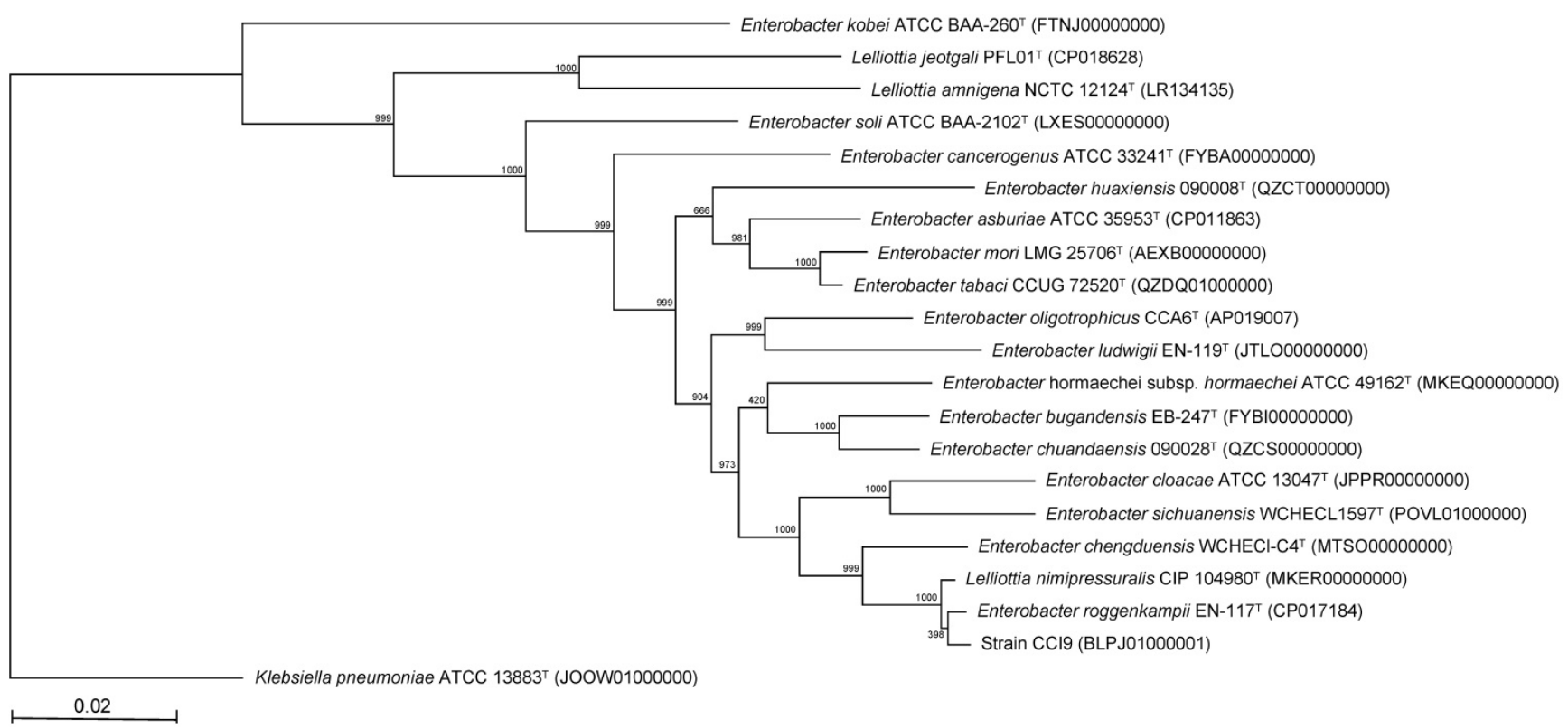

Figure 2. Phylogenetic tree constructed from analysis of the sequences of four housekeeping genes ( $a t p D$, gyr $B$, infB, and $r p o B)$ and showing the relationships between strain $\mathrm{CCl} 9$ and the related Enterobacter type strains. The bar indicates a $0.02 \%$ nucleotide substitution rate. The tree was rooted using Klebsiella pneumoniae aeruginosa ATCC $13883^{\top}$ as the outgroup.

Table 1. eggNOG categories of coding proteins from unique genes in $E$. roggenkampii $\mathrm{EN}-117^{\top}$ and $\mathrm{CCl}$

\begin{tabular}{|c|c|c|c|c|c|}
\hline \multirow[t]{2}{*}{ Class } & \multirow[t]{2}{*}{ Description } & \multicolumn{2}{|c|}{$\mathrm{EN}-117^{\mathrm{T}}$} & \multicolumn{2}{|l|}{ CCI9 } \\
\hline & & Count & Proportion (\%) & Count & Proportion $(\%)$ \\
\hline & Information storage and processing & & & & \\
\hline $\mathrm{J}$ & Translation, ribosomal structure, and biogenesis & 5 & 1.8 & 1 & 0.4 \\
\hline A & RNA processing and modification & 0 & 0 & 0 & 0 \\
\hline K & Transcription & 16 & 5.9 & 21 & 8.9 \\
\hline $\mathrm{L}$ & Replication, recombination, and repair & 38 & 13.9 & 33 & 14.0 \\
\hline \multirow[t]{2}{*}{ B } & Chromatin structure and dynamics & 0 & 0 & 0 & 0 \\
\hline & Cellular processes and signaling & & & & \\
\hline $\mathrm{D}$ & Cell cycle control, cell division, chromosome partitioning & 0 & 0 & 0 & 0 \\
\hline $\mathrm{Y}$ & Nuclear structure & 0 & 0 & 0 & 0 \\
\hline $\mathrm{V}$ & Defense mechanisms & 6 & 2.2 & 8 & 3.4 \\
\hline $\mathrm{T}$ & Signal transduction mechanisms & 1 & 0.4 & 6 & 2.5 \\
\hline M & Cell wall/membrane/envelope biogenesis & 12 & 4.4 & 29 & 12.3 \\
\hline $\mathrm{N}$ & Cell motility & 0 & 0 & 5 & 2.1 \\
\hline Z & Cytoskeleton & 0 & 0 & 0 & 0 \\
\hline W & Extracellular structures & 0 & 0 & 0 & 0 \\
\hline $\mathrm{U}$ & Intracellular trafficking, secretion, and vesicular transport & 3 & 1.1 & 4 & 1.7 \\
\hline \multirow[t]{2}{*}{$\mathrm{O}$} & Posttranslational modification, protein turnover, chaperones & 7 & 2.6 & 3 & 1.3 \\
\hline & Metabolism & & & & \\
\hline $\mathrm{C}$ & Energy production and conversion & 7 & 2.6 & 5 & 2.1 \\
\hline G & Carbohydrate transport and metabolism & 16 & 5.9 & 11 & 4.7 \\
\hline $\mathrm{E}$ & Amino acid transport and metabolism & 7 & 2.6 & 6 & 2.5 \\
\hline $\mathrm{F}$ & Nucleotide transport and metabolism & 2 & 0.7 & 1 & 0.4 \\
\hline $\mathrm{H}$ & Coenzyme transport and metabolism & 2 & 0.7 & 2 & 0.8 \\
\hline I & Lipid transport and metabolism & 2 & 0.7 & 2 & 0.8 \\
\hline $\mathrm{P}$ & Inorganic ion transport and metabolism & 3 & 1.1 & 1 & 0.4 \\
\hline \multirow[t]{2}{*}{$\mathrm{Q}$} & Secondary metabolites biosynthesis, transport, and catabolism & 1 & 0.4 & 3 & 1.3 \\
\hline & Poorly characterized & & & & 0 \\
\hline $\mathrm{R}$ & General function prediction only & 0 & 0 & 0 & 0 \\
\hline S & Function unknown & 145 & 53.1 & 95 & 40.3 \\
\hline
\end{tabular}


$\mathrm{EN}-117^{\top}$

CCI9

273

3918

236

Figure 3. Venn diagram of the shared and unshared genes in E. roggenkampii $\mathrm{EN}-117^{\mathrm{T}}$ and $\mathrm{CCl} 9$.

\section{Acknowledgments}

We thank Dr. Tatsuya Fujii for technical assistance with genome sequencing. We are grateful to all members of the Bio-conversion Research Group at our Institute [Research Institute for Sustainable Chemistry, National Institute of Advanced Industrial Science and Technology (AIST)] for their technical assistance and valuable discussions. This work was supported by a grant from JSPS KAKENHI to $\mathrm{H}$. Akita (19K15743).

\section{Competing Interests}

The authors have declared that no competing interest exists.

\section{References}

1. Raveendran S, Parameswaran B, Ummalyma SB, Abraham A, Mathew AK, Madhavan A, Rebello S, Pandey A. Applications of microbial enzymes in food industry. Food Technol Biotechnol. 2018;56:16-30.

2. Jozala AF, Geraldes DC, Tundisi LL, Feitosa VA, Breyer CA, Cardoso SL, Mazzola PG, Oliveira-Nascimento L, Rangel-Yagui CO, Magalhães PO, Oliveira MA, Pessoa A Jr. Biopharmaceuticals from microorganisms: from production to purification. Braz J Microbiol. 2016;47(S):51-63.

3. Sauer M, Porro D, Mattanovich D, Branduardi P. Microbial production of organic acids: expanding the markets. Trends Biotechnol. 2008;26:100108.

4. Lee SJ, Song H, Lee SY. Genome-based metabolic engineering of Mannheimia succiniciproducens for succinic acid production. Appl Environ Microbiol. 2006;72:1939-1948.

5. Sadh PK, Duhan S, Duhan JS. Agro-industrial wastes and their utilization using solid state fermentation: a review. Bioresour Bioprocess. 2018;5:1.

6. Ishida $\mathrm{Y}$, Kadota $\mathrm{H}$. Growth patterns and substrate requirements of naturally occurring obligate oligotrophs. Microb Ecol. 1981;7:123-130.

7. Tritt A, Eisen JA, Facciotti MT, Darling AE. An integrated pipeline for de novo assembly of microbial genomes. PLoS ONE. 2012;7:e42304.

8. Seemann T. Prokka: rapid prokaryotic genome annotation. Bioinformatics. 2014:30:2068-2069.

9. Huerta-Cepas J, Szklarczyk D, Heller D, Hernández-Plaza A, Forslund SK, Cook H, Mende DR, Letunic I, Rattei T, Jensen LJ, von Mering C, Bork P. eggNOG 5.0: a hierarchical, functionally and phylogenetically annotated orthology resource based on 5090 organisms and 2502 viruses. Nucleic Acids Res. 2019;47:D309-D314.

10. Goris J, Konstantinidis KT, Klappenbach JA, Coenye T, Vandamme P, Tiedje JM. DNA-DNA hybridization values and their relationship to whole-genome sequence simi-larities. Int J Syst Evol Microbiol. 2007;57:81-91.

11. Yoon SH, Ha SM, Lim J, Kwon S, Chun J. A large-scale evaluation of algorithms to calculate average nucleotide identity. Antonie van Leeuwenhoek. 2017;110:1281-1286.

12. $\mathrm{Xu} \mathrm{L}$, Dong $\mathrm{Z}$, Fang L, Luo $\mathrm{Y}$, Wei Z, Guo H, Zhang G, Gu YQ, Coleman-Derr D, Xia Q, Wang Y. OrthoVenn2: a web server for whole-genome comparison and annotation of orthologous clusters across multiple species. Nucleic Acids Res. 2019;47:W52-W58.
13. Brady C, Cleenwerck I, Venter S, Coutinho T, De Vos P. Taxonomic evaluation of the genus Enterobacter based on multilocus sequence analysis (MLSA): proposal to reclassify E. nimipressuralis and E. amnigenus into Lelliottia gen. nov. as Lelliottia nimipressuralis comb. nov. and Lelliottia amnigena comb. nov., respectively, E. gergoviae and E. pyrinus into Pluralibacter gen. nov. as Pluralibacter gergoviae comb. nov. and Pluralibacter pyrinus comb. nov., respectively, E. cowanii, E. radicincitans, E. oryzae and E. arachidis into Kosakonia gen. nov. as Kosakonia cowanii comb. nov., Kosakonia radicincitans comb. nov., Kosakonia oryzae comb. nov. and Kosakonia arachidis comb. nov., respectively, and E. turicensis, E. helveticus and E. pulveris into Cronobacter as Cronobacter zurichensis nom. nov., Cronobacter helveticus comb. nov. and Cronobacter pulveris comb. nov., respectively, and emended description of the genera Enterobacter and Cronobacter. Syst Appl Microbiol. 2013;36:309-319.

14. Brady C, Cleenwerck I, Venter S, Vancanneyt M, Swings J, Coutinho T. Phylogeny and identification of Pantoea species associated with plants, humans and the natural environment based on multilocus sequence analysis (MLSA). Syst Appl Microbiol. 2008;31:447-460.

15. Tamura K, Nei M. Estimation of the number of nucleotide substitutions in the control region of mitochondrial DNA in humans and chimpanzees. Mol Biol Evol. 1993;10:512-526.

16. Ohta H. Growth characteristics of Agromonas oligotrophica on ferulic acid. Microbes Environ. 2000;15:133-142.

17. Ohta H, Taniguchi S. Growth characteristics of the soil oligotrophic bacterium Agromonas oligotrophica JCM 1494 on diluted nutrient broth. J Gen Appl Microbiol. 1988;34:349-353.

18. Sutton GG, Brinkac LM, Clarke TH, Fouts DE. Enterobacter hormaechei subsp. hoffmannii subsp. nov., Enterobacter hormaechei subsp. xiangfangensis comb. nov., Enterobacter roggenkampii sp. nov., and Enterobacter muelleri is a later heterotypic synonym of Enterobacter asburiae based on computational analysis of sequenced Enterobacter genomes. F1000Res. 2018;7:521.

19. Jang JW, Jung HM, Im DK, Jung MY, Oh MK. Pathway engineering of Enterobacter aerogenes to improve acetoin production by reducing by-products formation. Enzyme Microb Technol. 2017;106:114-118.

20. Zhang L, Liu Q, Ge Y, Li L, Gao C, Xu P, Ma C. Biotechnological production of acetoin, a bio-based platform chemical, from a lignocellulosic resource by metabolically engineered Enterobacter cloacae. Green Chem. 2016;18:1560. 